Journal of Voice. Accepted for publication on 11/25/2015.

http://dx.doi.org/10.1016/j.jvoice.2015.11.021

\title{
Encouragement to increase the use of psychosocial skills in the di- agnosis and therapy of patients with functional dysphonia.
}

Jürg Kollbrunner, Ph.D.; Eberhard Seifert, M.D.

Division of Phoniatrics, Department of Otorhinolaryngology, Head Neck Surgery, University of Berne, Switzerland

E-mail correspondence: j.kollbrunner@bluewin.ch

Summary: Clinicians believe that psychosocial factors play a causal role in the etiology of many forms of functional dysphonia (FD). But for decades, all attempts to confirm such causation have failed. This paper aims to show the logic of this failure, to discuss the possibilities of employing psychology in therapy nonetheless, and to encourage clinicians to use their psychosocial knowledge and skills. The failure to confirm psychic and social factors as causal in the etiology of FD is basically a consequence of a principal shortcoming of evidence-based medicine (EBM). As the gold standard for validity, reliability, and objectivity in medical research, EBM is based on calculability and hence the processing of quantitative data. But life paths and life situations are best or sometimes only expressible in qualitative, experiential, and idiographic terms. Thus EBM-guided evaluation undervalues most psychosocial studies. This report of an experienced multidisciplinary voice team proposes alternative pathways for integrating psychosocial knowledge into the diagnosis and the treatment of FD. The difference between the fields of activity of psychotherapists and speechlanguage pathologists discussed, and the latter group is shown the potential benefits of using more of their psychosocial knowledge and skills.

Key Words: Functional dysphonia-Psychosocial-SLP and psychotherapeutic techniques-Conflict over speaking out-Morbid gain-Harmony and aggressivity-Guilt and shame. 


\section{Introduction}

Functional dysphonia (FD), characterized by hoarseness and/or reduced voice strength and/or disturbing laryngeal sensations in the absence of a structural or a neurobiological abnormality, is a common voice disorder ${ }^{1,2}$ appearing in different forms such as hypo- or hyperfunctional dysphonia, muscle misuse dysphonia (MMD), muscle tension dysphonia (MTD), puberphonia, psychogenic dysphonia, or conversion aphonia. Up to a third of patients suffering from a voice disorder referred to a multidisciplinary voice clinic receive the diagnosis of FD. ${ }^{2,3}$ Some forms of FD, namely psychogenic dysphonia and conversion aphonia, are clearly recognized as primarily caused by psychosocial processes. But what about the majority of FDs, in which, as in other "medically unexplained symptoms," psychosocial factors seem to play an important role? Are hypo- or hyperfunctional dysphonia, MMD, and MTD also caused by psychosocial processes?

\section{The search for psychosocial causes of FD}

In an attempt to answer this question, dozens of scientific investigations have been conducted gathering hundreds of results, mostly with positive findings concerning the psychosocial differences between patients with FD and controls without dysphonia. Some of the moderate and the minor psychosocial problems that have been identified as discriminating are:

perceived stress, traumatic stress experiences, mood disorders, depression, burnout, anxiety disorder, fear in social situations, emotional maladjustment, difficulty in the processing of negative emotions, difficulty dealing with anger, somatic preoccupation, excessive somatic complaints, somatization, neurosis, and clinical impression of hysteria. ${ }^{2,4-9}$

Some of the identified personality characteristics, several measured by the Minnesota Multiphasic Personality Inventory, are:

temperament, extraversion, introversion, neuroticism, type A personality (ambitious, rigidly organized), type $D$ personality (negative affectivity), social inhibition, social alienation, social competence, loneliness, interpersonal sensitivity, self-confidence, perfectionism, diffuse anxiety, control of emotions, impulsivity, suspiciousness, obsessivecompulsive trait, coping style, pessimism (dissatisfaction, sadness), and tendency toward denial. ${ }^{4,5,10-17}$ 
In recent years, evidence has been found that FD is at least correlated with depressive symptoms and general anxiety, ${ }^{5,18-21}$ but probably also with high neuroticism and hypochondriasis. ${ }^{1,4}$ But correlation does not imply causation. The discriminating findings could be causes of FD, but they could also be consequences of FD, or both could be covariables of a third factor. The experiences of clinicians often suggest the causality of psychological elements in the development of FD, but despite countless efforts to clarify this relation, no proof has been found. So specialists state: "The etiology of FD is still unclear," "18 "the pathogenic mechanisms are still far from being understood," 11 and "the interaction between predisposed and causal factors is unknown." 21

There are two possible explanations for this unsatisfactory state of research: Either the sought-after causalities do not exist or the search methods used are inappropriate.

Arguments against the first assumption include the experiences of clinicians but also the findings of modern psychoendocrinology, epigenetics, and neuroplasticity research, which all show striking causal relations between emotional states (e.g., loneliness, bereavement, or suppressed anger) and physical illness. The validity of the second assumption is hinted at by the fact that experimentation with human feelings and private thoughts are (fortunately) subject to severe humanitarian limitations. But experimentation is the crucial methodology in modern evidence-based medicine (EBM) and evidence-based practice (EBP). Is it possible that the characteristics and dominance of EBM and EBP are impeding the proof of the causality of psychosocial factors in the onset of FD?

\section{Are EBM and EBP obstacles in the search of psychosocial etiological factors?}

Since the 1990s, the development of EBM has seen a significant increase in the validity, reliability, and objectivity of medical research. The selection of strict criteria for evidence made it much easier to distinguish valid and reliable research findings from less useful results. The success of EBM, especially in the development of pharmaceuticals, has become so convincing that its criteria have become the gold standard. EBM as "the best tool to validate clinical decisions about the care of individual and aggregate patients" 22 "has meteorically emerged to dominate contemporary medical methods and practice". ${ }^{23}$ EBM is based on calculability, that is, on the processing of quantitative data. Its ideal is the randomized, controlled, double blinded, placebo- 
matched multicenter trial. But EBM methods cannot be applied to every research question. It is unable to process qualitative, phenomenological, experiential or idiographic data because "no mathematically driven algorithm will ever suffice for clinical reasoning," as Montgomery and Turkstra assert. ${ }^{24}$ EBM invokes instrumentalism, decontextualization and reductionism. ${ }^{23}$ Therefore, EBM judges the findings of studies that provide soft and subjective data as "of little or no evidence" and therefore as untrustworthy. So in reviews that search databases of potentially relevant studies, the majority of studies are regularly excluded from further analysis because uf insufficient compliance with EBM criteria. For instance, in their review of the effects of FD therapies, Ruotsalainen et al. ${ }^{25}$ searched seven databases using 100 keywords and found 5,937 papers on the treatment of FD and prevention of voice disorders. After five steps of "cleaning" (i.e., the elimination of EBM-incompatible studies) only seven papers remained for their meta-analysis. So hundreds of partially valued studies were declared to provide "minimal evidence" and became ignored. Conversely, the findings declared by EBM as "of strong evidence" are sometimes so anemic that they seem to be of little value. The low use of evidence-based findings in clinicians' decisionmaking speaks a clear language: Chan et al. ${ }^{26}$ surveyed 58 Australian speechlanguage pathologists (SLPS) about their use of EBP when treating adults with FD. A total of $98 \%$ of respondents reported that they relied on clinical experience to guide their clinical decision-making. Similarly, a survey of 240 American SLPs revealed that they use clinical experience and opinions of colleagues more frequently than research studies to guide their decisions. ${ }^{27}$ An online survey of 2,726 SLPs in 28 states showed that $91 \%$ had no scheduled time to support EBP activities. The majority of SLPs posed and researched zero to two EBP questions and read zero to four American Speech-Language-Hearing Association journal articles per year. ${ }^{28}$

In addition, the supremacy of EBP has serious general implications for the diagnosis and therapy of FD:

- In the absence of approved facts of inclusion, an FD diagnosis can only be given via exclusion, that is, if no organic reason can be found for the hoarseness, it must be (can be) FD. This makes FD "an undetected organic dysphonia," which contradicts the definition of FD.

- In the absence of approved causality-related aims for therapeutic change, the success of therapy is measured almost exclusively on the level of behavioral change. 
- The efficiency of EBM in many areas of scientific knowledge increases the unrealistic hope that the disorder will soon be recognized as of organic origin.

This unprovability via EBM keeps possible psychosocial causes of FD hidden. For although many research methods to identify psychosocial causal relations exist, and are widely used in the science of history, art, and literature, they have fallen out of favor in medical science. In contrast to the quantitative methods of nomothetic research strategies, these are the qualitative methods of idiographic research. In the nomothetic approach, large groups are investigated to find general laws of experience and behavior that apply to most people. The idiographic approach focuses on the individual; case studies, participant observation, narrative or in-depth interviews, qualitative content analysis, and semiotic data analysis are just some of the idiographic instruments. The idiographic approach takes the quality of research criteria just as seriously as the nomothetic approach, but the labels and meanings are slightly different. For example, the nomothetic concept of "validity" is replaced by the idiographic notion of "trustworthiness."

\section{Why psychosocial causation emerges only in idiographic research approaches} Knowledge of isolated psychological adjectives describing a person at a certain point in time, investigated by questionnaires (characteristics of personality, psychopathology, styles of attachment, coping strategies, or employment or family conflicts) is hardly sufficient for understanding a patient's life situation. For one patient (or his or her family) an increased degree of aggressiveness may be a major problem, whereas for another it might be a helpful way to show resistance in a manipulative social environment. Increased depression scores may be a sign of cautiousness and reservation in one patient but part of a deep inner emptiness in another. The complex of problems that is really stressing the patient only becomes comprehensible when it can be recognized in the context of the time frames of his or her development (Figure 1). The history lines through the time frames (e.g., at $\mathrm{t}^{1}=3$ years, $\mathrm{t}^{2}=16$ years, and $\mathrm{t}^{3}=$ present) allow the meaning of the characteristics in the individual frames to be understood. 


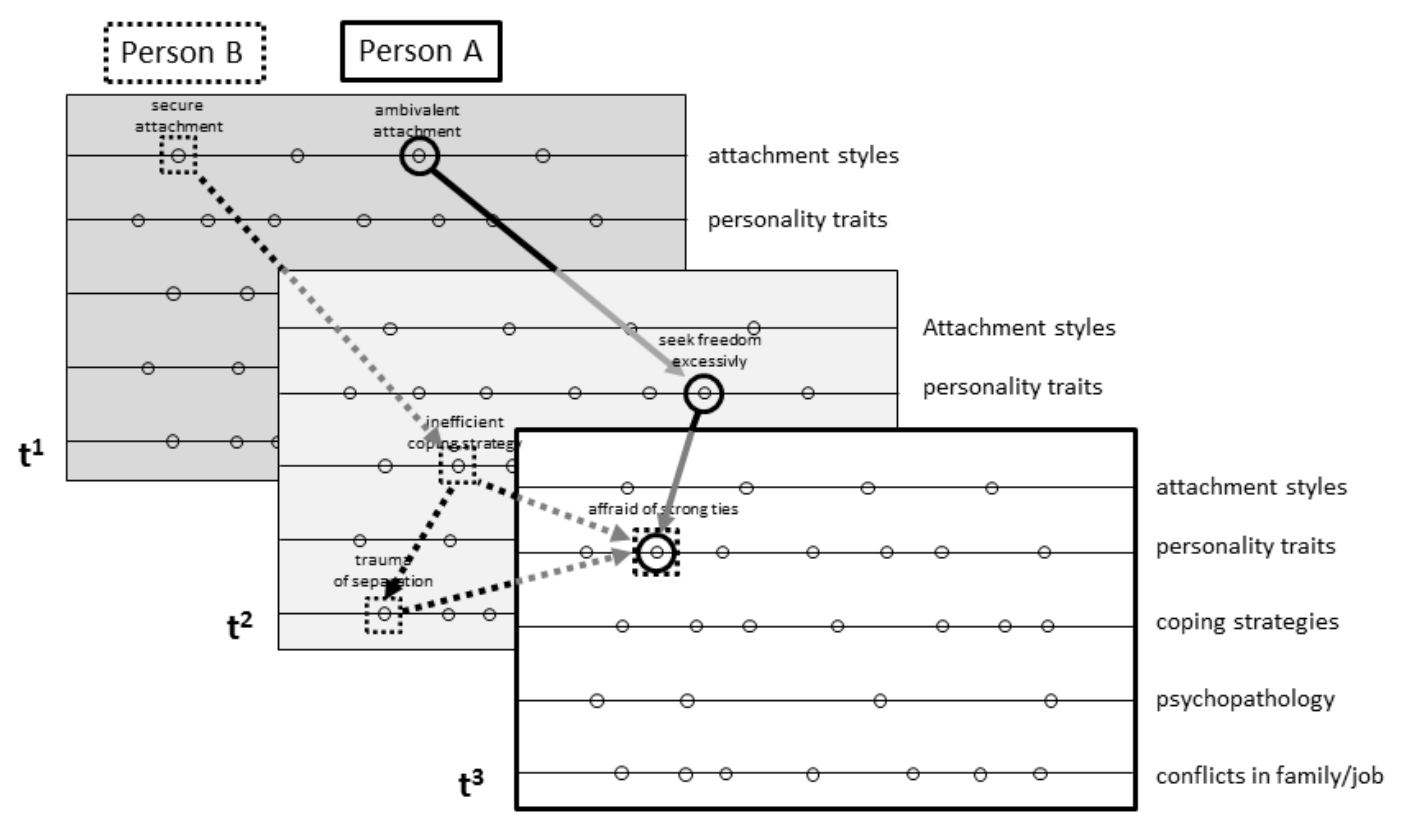

Fig. 1 Psychodiagnostik at different points in time

- attachment styles =e.g. "secure», "ambivalent» or "anxious-avoidant»
- personality traits =e.g. "extraversion» or "openness to experiences»
- coping strategies = e.g. "appraisal-focused» or "emotion-focused»
- psychopathology =e.g. "depression» or "obsessive disorder»
- conflict in family/job =e.g. "overprotection» or «mobbing»

An ambivalently attached child (person A) might later, as an adolescent, look for excessive freedom to prevent from being "swallowed" by others. Thus, as an adult, he or she will presumably be afraid of any strong interpersonal ties. A patient with this background must be seen differently from a patient (person B) who is also afraid of strong interpersonal ties, has grown up in a secure attachment, but who has only learned ineffectual coping strategies and who underwent a trauma of separation in his or her adolescence. A Deeper knowledge of the patient's path in life lets the function of a "functional disorder" (the morbid gain) appear more clearly. Is it used as dissociation, for protest, as a plea for attention, to avoid responsibility, as hidden execution of power, or some combination of such factors?

\section{Signs of a change}

Although there is no expectation that the dominance of EBP in evaluating research outcomes will decrease over the next decade, psychosomatic thinking is gaining ground. Ullas et al., ${ }^{29}$ British otorhinolaryngologists, write: "The treatment of medically unexplained symptoms has changed in recent years; there is now more emphasis on psychological factors due to an association with anxiety and depression." The discovery of the significance of the common factors in all types of therapy 20 years ago 
has promoted this development in an unexpected way: In a review of 17 metaanalyses comparing the outcome of different psychotherapeutic techniques, Luborsky et al $^{30}$ reported that the effects of psychotherapy seem to be primarily due to factors common to all therapies; no particular type of therapy can be shown to be significantly better than any other. Wampold et al. ${ }^{31}$ confirmed in a meta-analysis of 18 empirically validated therapy techniques that the different types of therapy showed almost zero difference in the extent of their effects. Wampold ${ }^{32}$ estimates that the variance in psychotherapy outcomes is due to the following factors:

- Common factors that underlie all psychotherapies, especially the therapeutic alliance: $>70 \%$,

- Specific effects of a particular treatment: $<8 \%$ and

- Client differences: $22 \%$.

In 2011, a joint task force from the American Psychological Association again confirmed the overwhelming significance of the therapeutic relationship, which is based on empathy, positive regard, and congruence (genuineness) on the part of the therapist. $^{33}$ There is no identifiable reason why the circumstances should be different in the therapy of FD: As soon as psychological factors play a crucial role, the therapeutic relationship becomes the principal therapeutic instrument. Some of today's SLPS recognize the deep significance of this: Millard and Cook ${ }^{34}$ declare that therapy itself is likely to be less important than the therapist and that a "therapeutic alliance" in an empathic and non-judgmental view of the client's world is crucial. Plexico et al. ${ }^{35}$ assert that "the qualities of the clinician, rather than specific therapy ingredients, are critical for a successful treatment outcome."

The therapeutic relationship is the key to developing the appreciation of psychosocial factors in patients with FD as well. It is only in a well-developed therapeutic alliance that the history of the patient with a functional disorder can be recognized within the time frames. In addition, "functional dysphonia has to be psychodiagnostically differentiated" ${ }^{21}$ and "effective voice therapy needs to include the management of psychological well-being." 20 This enhancement of psychosocial orientation and formation is possible under the basic principles of most different schools of psychotherapy. ${ }^{36}$ If learned in a responsible way, therapeutic techniques of cognitive behavioral therapy (CBT), psychodynamic therapy, systemic family therapy, or humanistic therapy can be used. 


\section{Which patients need a deeper appreciation of their psychosocial background?}

Some patients with FD do not need a deeper appreciation of their psychosocial background. This is particularly true of patients with MMD or MTD, the largest group, who often want nothing more than speedy recovery from their suffering. This is also true for those with a clear identifiable chronic professional vocal strain (although some, e.g. teachers, often benefit from recognizing and modifying the psychosocial factors that led to the chronic vocal strain). The voice specialist can propose a joint view of the patient's life situation, but the patient decides. Many other proven voice therapy techniques and counseling for vocal hygiene are available. Nevertheless, a deeper appreciation of a patient's psychosocial background is recommended, not only in cases of psychogenic dysphonia and conversion aphonia, but also in cases of MMD and MTD where a psychosocial component is appreciable behind the disorder. It should also be tried in cases of inexplicable therapeutic failure.

\section{Pathways to understanding the history and the life situation of patients with FD}

Exploring a patient's medical history must be more than the passive receipt of answers to a corresponding list of questions. It should result from an interaction that is controlled by the patient as much as by the clinician. Of course the clinician must ask for information in a structured way that allows him or her to understand the medical problem at hand. But sometimes the subjective meaning of a patient's suffering becomes recognizable only after the patient deviates for some time onto an unexpected topic.

The appreciation of the patient and his or her functional disorder will deepen if the clinician adopts some possible theories, insights and helpful attitudes as to the development of the FD and for therapy. Based on their experience, the multidisciplinary team from the University of Berne's Division of Phoniatrics proposes the following items:

- FD is the "intonation of a crisis": The disorder appears near the change from one phase of the individual's development to another and implies potentially paralyzing antagonistic forces; a struggle between the chance of advancement and the fear of its risks. Abresch ${ }^{37}$ alludes to the salutogenic function of the disorder. As the "intonation of a crisis", the voice disorder offers shelter from the conscious experience of frightening conflicting emotions. 
- FD as a cumulative trauma: Most patients with FD are not afflicted by a severe psychological illness or dramatic trauma. Often they live with high levels of stress or in some form of loneliness and passivity, have confusing interpersonal relationships, carry a burden of responsibility, or feel powerless. ${ }^{38}$ At times, it is hard for others to understand why these patients are affected so intensely by problems that seem normal to them. Often the answer is to find a disturbance in their early development of coping mechanisms. The same small trauma, experienced repeatedly over months or years (e.g., that their parents did not react sensitively to the expression of anger, joy, or anxiety) impeded their development of coping mechanisms.

- FD as a "momentary best solution": Vocal symptoms are a form of adaptation, a way of coming to terms with the environment, a method of merging inner drives and outer reality. ${ }^{39}$ The symptom is a vehicle for the patient until he or she has sufficient resources available to try other solutions. ${ }^{40}$

- FD as a morbid gain: Every functional disorder also has a "discomfort-reducing function," a sort of "hidden dividend." 39 Three forms can be differentiated: The primary gain serves to distract the patient from recognizing the frightening emotional conflict in his or her life situation. The secondary gain brings the patient attention, sympathy, protection, and relieves him or her of everyday duties. A tertiary gain can arise when parents or significant others benefit from the disorder (e.g., when a family member, now needing to care for the patient, will not be left alone). Morbid gain can become so fruitful "that some patients with functional voice disorders actually prefer dysphonia to a normal voice." ${ }^{41}$

- FD as "conflict over speaking out" (COSO) ${ }^{42}$ : FD is a product of inhibited emotional expression. It appears as "the silent or unuttered ' $\mathrm{No}^{\prime \prime}{ }^{40}$, an inability to set limits on one's interactions with others, regulated by the fear of being "punished" with refusal or to seem ungrateful and egoistic. Accordingly, the functional voice disorder is also "a price to pay for personal wishes that could not be directly expressed." ${ }^{39}$ The helpful attitudes are:

- Considering multiple generations: Understanding of the FD patient's life situation and history markedly increases if the clinician invites the patient to talk about his or her life story, perhaps via questions like: "Do you remember your voice as a child?", "What was your childhood like?" or "What is the current relationship with your parents?" Information about current and past conflicts and signs of vulnerability, resili- 
ence, and coping strategies are of interest (see Fig. 1) not simply as facts but as a mirror for the feelings of the patient. Goldstrohm ${ }^{43}$ writes:

"The history is obtained with emphasis on how the expression of emotionally charged ideas was accepted in the family of origin and in adult life, at work, or in the family of procreation."

- Acceptance of the relationship between harmony and aggression: Most individuals and families desire to live in peace and harmony. But the development of such a joyful emotional climate cannot emerge from affability and tenderness alone. It is not accessible without struggle and distress. Harmony is a by-product of tackling the problems life throws up; it cannot be achieved immediately. This topic can be brought up via questions like: "In what situations do you find yourself arguing?" or "In your family, who wins the most debates?"

- Attentiveness to remarks about guilt and shame: Some clinicians unhelpfully advise patients to "remove" unmotivated feelings of guilt and blame. But feelings are never unmotivated, and they cannot be commanded. At most they can be hidden. Feelings of guilt are often excruciating, but they can also be markers for "learned guilt" in childhood (which can be dismissed as an adult) or for "real guilt" (which can be accepted and a help one behave more thoughtfully).

- Monitoring of transference and countertransference: Transference, the unconscious redirection of feelings for or against a person in the past toward the therapist, should be carefully observed. It often explains a fractious style of cooperation with the therapist or an exaggerated compliance. Countertransference, the therapist's projection of his or her unresolved conflicts onto the patient, should be monitored equally carefully. It can be a sign that the therapist is misusing the patient to satisfy his or her own needs. Undetected, this is a real danger that can pervert the aims of therapy. The most effective prevention against this danger is regular supervision.

\section{Should SLPs be allowed to use techniques similar to psychotherapy?}

The background given above could suggest that patients with FD should generally be treated by psychologists or psychiatrists. But this reasoning is premature. Such a strategy would ignore the needs and the special scope of patients with FD.

The notion that SLPs could increase the application of their psychosocial knowledge and skills seems problematic. They are not psychotherapists, do not know the bene- 
fits and risks of psychosocial counseling, and may generate problems between the SLP and the psychology associations. But such a conflict also offers an opportunity: The extension of the SLPs' skills with psychotherapeutic techniques could break traditional therapeutic barriers to harness the healing power of the therapeutic relationship, exactly as Travis, a founding father of SLP in America, proposed 60 years ago ${ }^{44}$ when he claimed that within a few years speech therapists would successfully apply the principles and techniques of psychotherapy. Let us look at this in detail. For most patients, it is painful to suffer from a condition that has partly psychological roots. In the case of patients with FD, this aversion often has a special function as a second type of primary morbid gain. The unconscious development of a physical symptom (instead of a psychological one) enables them to consolidate their distance from the frightening conflict. The SLPS' wide physiological knowledge of voice production makes them exactly the right person to show the patient profound interest in their distress, and to lead them softly from the foreground of their symptom to the background of their voice disorder. The fact that many patients with FD were proposed therapy with a psychologist or psychiatrist but never followed through indicates the importance of this unique possibility.

Of course patients with FD with major psychopathological problems must be allocated to a psychotherapist or a psychiatrist. But most patients with FD are afflicted with only minor=psychopathological problems. For these patients an SLP, experienced in voice pathology, voice therapy, and the psychosocial techniques of assistance, is the most appropriate professional.

Unfortunately, in their basic education SLPs learn little about psychodynamic interrelations and how to handle them. To overcome this, Scott et al. ${ }^{1}$ suggest:

"Because the psychological skills of speech therapists are acquired in a haphazard fashion, it is advocated that clinical psychologists develop a training and consultative role for speech therapy, equipping therapists with additional psychological skills, leading to more effective treatment for patients with functional dysphonia.

Miller et al. ${ }^{20}$ add:

"Allied health professionals are increasingly being trained to use CBT skills in the management of a number of symptoms/illnesses, and this should be considered for the management of functional dysphonia." 
Voice scientists from British and Australian universities are currently conducting a large research project to improve training for voice therapists in psychosocial thinking and handling. ${ }^{45}$

\section{Consequences for well-skilled but psychologically less-trained SLPs}

The assertion of some SLPs that they - as responsible professionals - should never try to apply techniques similar to psychotherapy because these are reserved for fully trained psychotherapists is not cogent. SLPs always use interventions similar to psychotherapeutic ones; they cannot prevent this. In the manner of the famous quote by Paul Watzlawick, we must say: "It's not possible to act therapeutically without acting psychotherapeutically." For good or ill, it must be added. Often therapists are not aware that seemingly ordinary verbal reactions can lead to substantial psychotherapeutic effects on the patient's experience. An example:

A 30 year-old-woman with FD says:

"I could explode when my mother still tells me how I should dress over the phone." The SLP can answer:

(1) "You should defend yourself." or

(2) "Don't take this so seriously; ignore it." or

(3) "Did you tell her that you don't want to hear such advice?" or

(4) "Yes, fashion has changed since twenty years ago." or

(5) "Are there other situations where your mother makes you angry?" or

(6) "I understand that this makes you indignant." or

(7) "My mother behaved exactly the same way." or

(8) "Are there other demands and expectations from your mother?" or

(9) "When you see that your mother is calling, you could simply let the phone ring."

Answers (3), (5), and (8) are more appropriate than, for example, (2) (disrespecting the patient's anger) or (7) (a reaction based on clinician's countertransference).

The example shows that it's really better when SLPs enlarge and deepen their psychosocial knowledge and skills (even if only slightly), than to pretend they never influence the feelings and thoughts of their patients.

Nevertheless, SLPs are often conservative in their application of psychosocial skills. A strong sense of responsibility lets them avoid interventions that seem too similar to psychotherapeutic techniques. They consider them too dangerous and fear the reactions they could trigger with an intervention similar to psychotherapy; they worry they 
will not be able to handle the emotions that arise or solve the patient's newly emerging problems. But such situations are not really dangerous. If the SLP is prepared to stay in responsible contact with mindfulness but without acting hastily, nothing needs to be "done" on most of these occasions. Normally, the intense emotions that arise are very familiar to the patient and it is beneficial for him or her to display them without being evaluated. Likewise, new reported problems are mostly all too familiar for the patient and the clinician has no obligation (and no way) to solve them for the patient. But by reporting on them to a trustworthy equal, the patient creates a chance to hear new perspectives in his own vocalized words and in the clinicians' reactions. The dangers in therapeutic relationships lie elsewhere. Fundamentalist dogmatic therapists, that is, "allies" who permanently try to press an opinion or behavior on the patient are dangerous, particularly when they appear in a gentle form as an amiable guide to the "right way." Clinicians who are in mindful contact with their patients will never force them in a particular direction.

The presented pathways with usable theories, insights and attitudes can still be a source of hesitation. SLPs may claim that, even when equipped with psychosocial skills, they are not able to consider all of this and carry out full psychotherapeutic diagnosis and treatment. But they do not to have to imitate psychotherapists, complete elaborated psychodiagnostic surveys, or treat neuroses or even deeper psychopathology. The simple fact is that the psychosocial factors given above exist. They can be heeded or ignored. By paying attention to them, SLPs can suggest looking together with their patients at certain patterns in their patient's current situation or history, constellations that could be associated with or even causal to their disorder. The patient will quickly demonstrate whether he or she accepts this suggestion or not. If he or she does, the SLP and the patient are already together on a journey, searching for alternative ways of thinking, interpreting feelings and behavior in situations in which the patient feels agitated or blocked. The subject of such a journey is chosen by the therapist. From the various facts, thoughts, and feelings the patient reports, the clinician can choose the topic (associations between some of the reported elements) he thinks will best deepen a mutual understanding of the patient and his or her disorder. The number of issues broached in an individual therapeutic session - perhaps one to three - is unimportant, because most relevant personal subjects are interrelated. The aim of such a (psycho-)therapeutic journey is to support the patient in his or her self-awareness and self-assurance by assisting him or her in 
the search for more satisfying ways of thinking, feeling, and behaving. Often no advice need be given, because most patients are quick to recognize their own ability to cope as soon as they begin to believe that these capacities really dwell in them and can be harnessed. Several psychologists encourage SLPS to enlarge their psychosocial skills: Kriz ${ }^{46}$ speaks of expanding SLPs' thoughts and actions in the direction of psychotherapy, and Wolfart ${ }^{47}$ advocates a joint therapeutic culture for SLPs and psychotherapy.

However, a strict requirement for SLPs adopting a psychosocial focus is regular participation in supervision guided by a psychotherapist. In this journey, puzzling, painful, or seductive situations in therapy can be analyzed in the context of the history of the client and the clinician. This can identify and mitigate potentially dangerous countertransference.

\section{References}

01 Scott S, Deary IJ, Mackenzie K, Wilson JA. Functional dysphonia: a role for psychologists? Psychol Health Med. 1997;2(2): 169-180.

02 Roy N. Functional dysphonia. Curr Op Otolaryngol Head Neck Surg. 2003;11: 144-148.

03 Bohlender J. Diagnostic and therapeutic pitfalls in benign vocal fold diseases. GMS Curr Top Otorhinolaryngol Hed Neck Surg. 2013;12: 1-18.

04 Roy N, McGrory JJ, Tasko SM, Bless DM, Heisey D, Ford CN. Psychological correlates of functional dysphonia. An investigation using the Minnesota Multiphasic Personality Inventory. J Voice. 1997;11(4): 443-451.

05 Lauriello M, Cozza K, Rossi A, Di Rienzo L, Coen Tirelli G. Psychological profile of dysfunctional dysphonia. Acta Otorhinolaringol Ital. 2003;23: 467-473.

06 Baker J. The role of psychogenic and psychosocial factors in the development of functional voice disorders. Int J Speech Lang Pathol. 2008;10(4): 210-230.

07 Baker J. Women's voice: lost or mislaid, stolen or strayed? Int J Speech Lang Pathol. 2010;12(2): 94-106

08 Siupsinskiene N, Razbadauskas A, Dubosas L. Psychological distress in patients with benign voice disorders. Folia Phoniatr Logop. 2011;63: 281-288.

09 Reiter R, Rommel D, Brosch S. Long term outcome of psychogenic voice disorders. Auris Nasus Larynx. 2013; 40: 470-475.

10 Winkler F. [Psychogenic voice disorders. Contribution of clinical psychology]. [In German]. HNO. 1987:35(6): 242-245.

11 Andersson K, Schalén L. Etiology and treatment of psychogenic voice disorder: Results of a follow-up study of thirty patients. J Voice. 1998;12(1): 96-106. 
12 Roy N, Bless DM, Heisey D. Personality and voice disorders: A multitraitmultidisorder analysis. J Voice. 2000;14(4): 521-548.

13 Meulenbroek LFP, Thomas G, Kooijman PGC, de Jong FICRS. Biopsychosocial impact of the voice in relation to the psychological features in female student teachers. J Psychosom Res. 2010;68: 379-384.

14 Van Wijck-Warnaar A, Van Opstal MJ, Exelmans K, Schaekers K, Thomas G, de Jong FI. Biopsychosocial impact of voicing and general coping style in teachers. Folia Phoniatr Logop. 2010;62(1-2): 40-46.

15 O'Hara J, Miller T, Carding P, Wilson J, Deary V. Relationship between fatigue, perfectionism, and functional dysphonia. Otolaryngol Head Neck Surg. 2011;144(6): 921-926.

$16 \mathrm{Ng} \mathrm{JH}$, Lo S, Lim F, Goh S, Kanagalingam J. Association between anxiety, type A personality, and treatment outcome of dysphonia due to benign causes. Otolaryngol Head Neck Surg. 2013;148(1): 96-102.

17 Conture EG, Kelly EM, Walden TA. Temperament, speech and language: An overview. J Commun Disord. 2013;46(2): 125-142.

18 Willingera U, Völkl-Kernstock, Aschauer HN. Marked depression and anxiety in patients with functional dysphonia. Psychiatry Res. 2005;134(1): 85-91.

19 Dietrich M, Verdolini Abbott K, Gartner-Schmidt J, Rosen CA. The frequency of perceived stress, anxiety, and depression in patients with common pathologies affecting voice. J Voice. 2008;22(4): 472-488.

20 Miller T, Deary V, Patterson J. Improving access to psychological therapies in voice disorders: a cognitive behavioural therapy model. Curr Opin Otolaryngol Head Neck Surg. 2014;22(3): 201-205.

21 Kiese-Himmel C. Clinical-psychological components in the consideration of functional dysphonia - a review [n German]. Laryngo-Rhino Otol. 2015;94(3): 156-162.

22 Beltran RA. Book Review: The Gold Standard: The challenge of evidence-based medicine and standardization in Health care. J Nat Med Ass. 2005;97(1): 110.

23 McMaster R. Beware those offering "Gold Standards": Evidence-based medicine and the potential for institutional change in clinical-medical provision. J Economic Issues; 2008;42(2): 885-912.

24 Montgomery EB, Turkstra L. Evidence-based medicine: Let's be reasonable. J Med Speech Lang Pathol. 2003;11(2): ix-xii.

25 Ruotsalainen J, Sellman J, Lic P, Lehto L, Verbeek J. Systematic review of the treatment of functional dysphonia and prevention of voice disorders. Otolaryngol Head Neck Surg. 2008;138: 557-565.

26 Chan AK, McCabe P, Madill CJ. The implementation of evidence-based practice in the management of adults with functional voice disorders: A national survey of speech-language pathologists. Int J Speech Lang Pathol. 2013;15(3): 334-344.

27 Zipoli RP, Kennedy M. Evidence-based practice among speech-language pathologists. Attitudes, utilization, and barriers. Am J Speech Lang Pathol. 2005;14: 208-220. 
28 Hoffman LM, Ireland M, Hall-Mils S, Flynn P. Evidence-based speech-languagepathology practices in schools: Findings from a national survey. Lang Speech Hear Serv Schools. 2013;44: 266-280.

29 Ullas G, McClelland L, Jones NS. Medically unexplained symptoms and somatisation in ENT. J Laryngol \& Otol. 2013;127: 452-457.

30 Luborsky L, Rosenthal R, Diguer L, Andrusyna TP, Berman JS, Levitt JT, Seligman DA, Krause ED. The dodo bird verdict is alive and well - mostly. Clin Psychol: Science and Practice. 2002;9(1): 2-12.

31 Wampold BE, Mondin GW, Moody M, Stich F, Benson K, Ahn H. A meta-analysis of outcome studies comparing bona fide psychotherapies: Empirically, "All Must Have Prizes". Psychol Bull. 1997;122(3): 203-215.

32 Wampold BE. Great psychotherapy debate: Models, methods, and findings. Mahweh: Taylor \& Francis; 2001.

33 Norcross JC, Wampold BE. Evidence-based therapy relationships: Research conclusions and clinical practices. Psychotherapy. 2011;48(1): 98-102.

34 Millard SK, Cook FM. Working with young children who stutter: raising our game. Sem Speech Lang. 2010;31(4): 250-261.

35 Plexico LW, Manning WH, DiLollo A. Client perceptions of effective and ineffective therapeutic alliances during treatment for stuttering. J Fluency Dis. 2010;35(4): 333-354.

36 Johnson GW, Gonzales CT, Chang CJ, Maines SL, Mainville MM, Asbury AJ. Psychogenic voice disorders. In: Nemecek O, Mares V (eds). Laryngeal diseases, symptoms, diagnosis and treatments. Hauppauge NY: Nova science Publishers; 2010: 1-28.

37 Abresch J. Stimmstörung als Krisenvertonung. Über biographische Einflüsse auf die Gewordenheit unserer Stimme und über die Entstehung funktioneller Stimmstörungen. Integrative Therapie. 1988;1: 40-62.

38 Butcher P. Psychological processes in psychogenic voice disorder. Eur J Disord Commun. 1995;30(4): 467-474.

39 Murphy AT. Functional voice disorders. Englewood Cliffs: Prentice Hall; 1964.

40 Paldele-Schöberl R. Funktionelle Aphonie - Funktionelle Dysphonie. Das stumme Nein. [unpublished diploma thesis]. Wien: SAB; 1997.

41 Van Lierde KM, Claeys S, De Bodt M, van Cauwenberge P. Long-term outcome of hyperfunctional voice disorders based on a multiparameter approach. $J$ Voice. 2007;21(2): 179-188.

42 House AO, Andrews HB. Life events and difficulties preceding the onset of functional dysphonia. J Psychosom Res. 1988;32(3): 311-319.

43 Goldstrom SL. The role of psychological factors in muscle tension dysphonia. 2012. http://www.ucsfcme.com/2012/slides/MOT12002/Finished\%20Voice \%20PDFs/19GoldstrohmThe RoleOfPsychologicalFactorsnMuscleTensionDysphonia.pdf. Accessed December 22, 2015.

44 Travis LE. The psychotherapeutical process. In: Travis LE (ed). Handbook of speech pathology. New York: Appleton-Century-Crofts; 1957: 965-990. 
45 Deary V. Training speech therapists in cognitive behavioural therapy to treat medically unexplained dysphonia: A trial platform. 2015 http://gtr.rcuk.ac.uk/projects? ref=G0501875. Accessed December 22, 2015

46 Ritterfeld U. Können Logopädinnen psychotherapeutisch arbeiten? Ein Gespräch mit dem Osnabrücker Psychologen und Psychotherapeuten Prof. Jürgen Kriz. Logos Interdiszipl. 1994;2: 132-138.

47 Wolfart M. Was verbindet, was trennt Logopädie und Psychotherapie? Forum Logop. 2001;15: 7-14. 